\title{
The Jebsen-Birkhoff theorem in alternative gravity
}

\author{
Valerio Faraon:* \\ Physics Department, Bishop's University \\ Sherbrooke, Québec, Canada J1M 1Z7
}

\begin{abstract}
We discuss the validity, or lack thereof, of the Jebsen-Birkhoff theorem in scalar-tensor theories by generalizing it and regarding the Brans-Dicke-like scalar as effective matter. Both the Jordan and Einstein frames are discussed and an apparent contradiction between static spherical solutions of scalar-tensor gravity and Hawking's theorem on Brans-Dicke black holes is clarified. The results are applied to metric and Palatini $f(R)$ gravity.

PACS numbers: $04.50 . \mathrm{Kd}, 04.20 . \mathrm{Cv}, 04.70 . \mathrm{Bw}$
\end{abstract}

\section{INTRODUCTION}

The recent attempts to explain the acceleration of the universe discovered with type Ia supernovae [1] have led to the introduction of an ad hoc dark energy comprising $76 \%$ of the energy content of the universe and with an extreme (possibly phantom) equation of state $P \simeq-\rho$, where $\rho$ and $P$ are the effective energy density and pressure of the dark energy 2]. Displeased with this explanation, many authors have turned to the possibility that dark energy does not exist and, instead, gravity must be modified in the infrared sector 3, 4] and the so-called $f(R)$ gravity theories have been resurrected for this purpose. $f(R)$ gravity theories in use in cosmology come in three versions, called the metric formalism [3, 4] , Palatini formalism [5], and metric-affine gravity [6] (see [7] for a recent review and [8] for shorter introductions). The study of spherically symmetric solutions has been crucial to understand the weak-field limit of these theories and confront them with Solar System observations (remember that the three classical tests of General Relativity are based on the spherically symmetric Schwarzschild solution [9, 10]). Morever, the study of strong field instabilities potentially fatal for the theory is carried out in spherical symmetry [11]. It is therefore important to understand how the Jebsen-Birkhoff theorem well known from spherical symmetry in General Relativity generalizes, or fails, in these extensions of Einstein's theory. Students beginning in General Relativity are familiar with Birkhoff's theorem stating that a spherically symmetric solution of the vacuum Einstein equations is static [13] (it appears [14-16] that this result had actually been discovered by Jebsen two years before Birkhoff [17]). It is now well-known that the Jebsen-Birkhoff theorem does not hold in metric $f(R)$ gravity while it is valid in Palatini $f(R)$ gravity 7]. Since both metric and Palatini $f(R)$ gravity admit a description as special scalar-tensor theories (Brans-Dicke theories with values of the Brans-Dicke parameter $\omega=0$ and $\omega=-3 / 2$, respectively, and endowed with a special potential for the Brans-Dicke scalar [7, 18]), a complete understanding of the Jebsen-Birkhoff theorem in $f(R)$ gravity requires its understanding in general scalar-tensor gravity, which is the goal of the present paper. Beginning with the realization that the Brans-Dicke-like scalar field of scalar-tensor gravity can, and most often is, regarded as an effective form of matter for effective Einstein equations, one is led to formulate a generalized Jebsen-Birkhoff theorem in the presence of matter in General Relativity, which is convenient for the study of spherical symmetry in scalartensor gravity (this is done in Secs. II and III using the Jordan frame). An alternative approach to scalar-tensor gravity consists of using its Einstein frame formulation, which is employed in Sec. IV. In this regard, an apparent contradiction between certain long-known exact spherical solutions of scalar-tensor theory 19, 21 and Hawking's theorem for Brans-Dicke black holes [22] is elucidated. Almost as a byproduct of this work, light is shed on the validity, or lack thereof, of the Jebsen-Birkhoff theorem of Palatini and metric $f(R)$ gravity in Sec. V.

We adopt the notations of Ref. [9]. The cosmological constant $\Lambda$, if present, is seen as an effective form of matter described by the formal stress-energy tensor $T_{a b}^{(\Lambda)}=-\frac{\Lambda}{8 \pi G} g_{a b}$ (that is, in the following, "vacuum" implies that $\Lambda=0$ ).

\section{THE JEBSEN-BIRKHOFF THEOREM IN GENERAL RELATIVITY}

The most general spherically symmetric line element can be written as

$$
d s^{2}=-A^{2}(t, r) d t^{2}+B^{2}(t, r) d r^{2}+r^{2} d \Omega_{2}^{2}
$$

where $d \Omega_{2}^{2}=d \theta^{2}+\sin ^{2} \theta d \varphi^{2}$ is the line element on the unit 2 -sphere and $r$ is an areal radius. The metric components $g_{0 i}(i=1,2,3)$, if present, can be eliminated by redefining the coordinates $t$ and $r$ ([17], see also [23]). The $(0,1),(0,0),(1,1),(2,2)$, and $(3,3)$ components of the Einstein field equations

$$
R_{a b}-\frac{1}{2} g_{a b} R=8 \pi G T_{a b}^{(m)}
$$


where $T_{a b}^{(m)}$ is the matter energy-momentum tensor, yield

$$
\begin{aligned}
& \frac{\dot{B}}{B}=4 \pi G r T_{01}^{(m)}, \\
& \frac{1}{r^{2}}+\frac{2 B^{\prime}}{B^{3} r}-\frac{1}{B^{2} r^{2}}=8 \pi G \frac{T_{00}^{(m)}}{A^{2}}, \\
& \frac{2 A^{\prime}}{A r}-\frac{B^{2}}{r^{2}}+\frac{1}{r^{2}}=8 \pi G T_{11}^{(m)}, \\
& \frac{r}{B^{3}}\left(\frac{A^{\prime} B}{A}-B^{\prime}-\frac{r B^{2} \ddot{B}}{A^{2}}+\frac{r \dot{A} B^{2} \dot{B}}{A^{3}}-\frac{r A^{\prime} B^{\prime}}{A}+\frac{r A^{\prime \prime} B}{A}\right) \\
& =8 \pi G T_{22}^{(m)}, \\
& \frac{r \sin ^{2} \theta}{B^{3}}\left(\frac{A^{\prime} B}{A}-B^{\prime}-\frac{r B^{2} \ddot{B}}{A^{2}}+\frac{r \dot{A} B^{2} \dot{B}}{A^{3}}-\frac{r A^{\prime} B^{\prime}}{A}\right. \\
& \left.+\frac{r A^{\prime \prime} B}{A}\right)=8 \pi G T_{33}^{(m)},
\end{aligned}
$$

. (3) with the assumption $J_{(r)}=0$ (equivalent to $T_{01}^{(m)}=0$ in a region where $A$ and $B$ are finite and positive) guarantees that $\dot{B}=0$, i.e., $B=B(r)$. Then eq. (5) with the assumption $\partial P_{(r)} / \partial t=0$ guarantees that $A^{\prime} / A$ is time-independent, which only leaves the possi-

(6) bility that $A$ depends on time through a multiplicative factor, $A(t, r)=f(t) a(r)$. But then the line element assumes the form

$$
d s^{2}=-a^{2}(r) f^{2}(t) d t^{2}+B^{2}(r) d r^{2}+r^{2} d \Omega_{2}^{2}
$$

where a prime and an overdot denote differentiation with respect to $r$ and $t$, respectively.

\section{The non-vacuum case}

Let us consider a timelike observer at rest in the coordinate system $(t, r, \theta, \varphi)$ adapted to the spherical symmetry of the metric (11), i.e., one with 4 -velocity $u^{\mu}=$ $\left(A^{-1}, 0,0,0\right)$. The matter energy density relative to this observer "at rest" is

$$
\rho \equiv T_{a b}^{(m)} u^{a} u^{b}=\frac{T_{00}^{(m)}}{A^{2}}
$$

and coincides with the right hand side of the Hamiltonian constraint (4) apart from the factor $8 \pi G$. The radial energy current relative to this observer is

$$
J_{(r)} \equiv-T_{a b}^{(m)} u^{a} e_{(r)}^{b}=\frac{T_{01}^{(m)}}{A B}
$$

where $e_{(r)}^{b}$ is the spacelike unit vector in the radial direction with components $e_{(r)}^{\mu}=\left(0, B^{-1}, 0,0\right)$. Finally, the radial pressure relative to this observer is

$$
P_{(r)} \equiv T_{a b}^{(m)} e_{(r)}^{a} e_{(r)}^{b}=\frac{T_{11}^{(m)}}{B^{2}}
$$

The non-radial stresses $T_{i j}^{(m)}$ with $i \neq j(i, j=1,2,3)$ vanish identically because of the Einstein equations and the fact that the components $G_{i j}$ of the Einstein tensor with $i \neq j$ vanish in spherical symmetry. Furthermore, eqs. (6) and (7) imply that $\frac{T_{33}^{(m)}}{\sin ^{2} \theta}=T_{22}^{(m)}$. and the redefinition of the time coordinate $t \rightarrow \bar{t}$ with $d \bar{t} \equiv f(t) d t$ then absorbs the factor $f(t)$ into $\bar{t}$ and casts the metric into locally static form. Then the Einstein equations (6) and (7) imply that also $T_{22}^{(m)}$ and $T_{33}^{(m)}$ are time-independent, and this is true for the tangential pressures

$$
\begin{aligned}
P_{(\theta)} & \equiv T_{a b}^{(m)} e_{(\theta)}^{a} e_{(\theta)}^{b}=\frac{T_{22}^{(m)}}{r^{2}} \\
& =P_{(\varphi)} \equiv T_{a b}^{(m)} e_{(\varphi)}^{a} e_{(\varphi)}^{b}=\frac{T_{33}^{(m)}}{r^{2} \sin ^{2} \theta}
\end{aligned}
$$

as well, where $e_{(\theta)}^{a}$ and $e_{(\varphi)}^{a}$ are spacelike unit vectors in the angular directions. We have therefore the

Jebsen-Birkhoff theorem (version 1): If a solution of the Einstein equations is spherically symmetric and the matter distribution is static $\left(\frac{\partial \rho}{\partial t}=\frac{\partial P_{(r)}}{\partial t}=0\right.$ and $\left.J_{(r)}=0\right)$, then the metric is static in a region in which

The restriction to the region where the coordinates preserve their timelike or spacelike character is necessary for the validity of the theorem, as originally noted by Ehlers and Krasinski [24]. For example, this restriction is not satisfied in the region inside the Schwarzschild black hole horizon or outside the de Sitter cosmological horizon, where these metrics become time-dependent. A better statement of the Jebsen-Birkhoff theorem is that, under the conditions stated "a spherically symmetric solution admits, besides the $\mathrm{SO}(3)$ generators, an additional hypersurface-orthogonal Killing vector field" ([24], see also [12, 25, 26]). However, the metric is static only where this additional Killing field remains timelike, which excludes the black hole horizon (if this exists and coincides with the Killing horizon) where the Killing field $t$ remains timelike and $(r, \theta, \varphi)$ stay spacelike. 
goes through zero to change sign in the interior [62]. The theorem does not make statements valid on a black hole horizon surface which may be present and it does not imply that the solution of the Einstein equations is the Schwarzschild metric. A solution with a matter distribution diverging on a horizon is possible; this happens, for example, for the Brans class I solutions of scalar-tensor gravity [19] (in which the Brans-Dicke-like scalar field can be regarded as a form of effective matter) which are static and spherically symmetric but have a scalar field diverging at the horizon-see Sec. IV.

It might seem that the assumptions on the matter $T_{a b}^{(m)}$ are too strong because this tensor contains the metric $g_{a b}$ and, therefore, any assumption on $T_{a b}^{(m)}$ means to require already, in some way, that the metric $g_{a b}$ is static. Indeed, this is true but the assumption of a static matter distribution leaves room for physically relevant situations. The first is vacuum, in which no-matter is necessarily (and trivially) of the static form and gives rise to the more familiar version of the Jebsen-Birkhoff theorem upon which we comment in the next subsection. Another non-trivial situation is that of a cosmological constant seen as a form of effective matter described by $T_{a b}^{(\Lambda)}$, which is spherically symmetric and static. In this case the solution is not the Schwarzschild but the Schwarzschild-(anti)de Sitter one. It is well known that, for example, the Schwarschild-de Sitter (Kottler) line element can be put in the locally static form

$$
\begin{aligned}
d s^{2} & =-\left(1-\frac{2 G M}{r}-\frac{\Lambda r^{2}}{3}\right) d t^{2} \\
& +\left(1-\frac{2 G M}{r}-\frac{\Lambda r^{2}}{3}\right)^{-1} d r^{2}+r^{2} d \Omega_{2}^{2}
\end{aligned}
$$

in the region of spacetime manifold between the black hole and the cosmological horizons. Another physical situation allowed by the assumptions of the theorem is electro-vacuum [27]. If the mass distribution carries a static electric charge $Q$ with no radial current the solution is the static Reissner-Nordstrom metric given by

$$
\begin{aligned}
d s^{2} & =-\left(1-\frac{2 G M}{r}+\frac{Q^{2}}{r^{2}}\right) d t^{2} \\
& +\left(1-\frac{2 G M}{r}+\frac{Q^{2}}{r^{2}}\right)^{-1} d r^{2}+r^{2} d \Omega_{2}^{2} .
\end{aligned}
$$

The absence of a radial energy current, $J_{(r)}=0$, without the assumption of staticity of $\rho$ and $P_{(r)}$ is not sufficient to guarantee that the metric is static. A counterexample is the McVittie solution describing a spherical object embedded in a cosmological background [28] for which $J_{(r)}=0$ but the metric is time-dependent except for the special case in which it reduces to the Schwarzschild-(anti)de Sitter one.

Although less familiar than the vacuum version, version 1 of the Jebsen-Birkhoff theorem is more suitable for discussing the spherically symmetric solutions of scalartensor gravity because the Brans-Dicke-like scalar field present in these theories acts as an effective form of matter.

\section{The vacuum case}

By regarding vacuum $\left(T_{a b}^{(m)}=0\right)$ as a form of static matter, the Jebsen-Birkhoff theorem assumes its most familiar form.

Jebsen-Birkhoff theorem (version 2): a spherically symmetric solution of the vacuum Einstein equations is necessarily static in a region in which $t$ remains timelike and $(r, \theta, \varphi)$ stay spacelike.

The vacuum assumption rules out the possibility of a cosmological constant as well as electro-vacuum, and the Schwarzschild-(anti)de Sitter and ReissnerNordstrom solutions. The solution is then forced to be Schwarzschild.

Note that the vacuum as defined by $T_{a b}=0$ is not necessarily a trivial configuration in alternative theories of gravity. In scalar-tensor gravity the Brans-Dicke-like scalar field $\phi$ describing the gravitational field together with the metric $g_{a b}$ may be non-constant and still give a vanishing effective stress-energy tensor $T_{a b}^{(\phi)}$; this is referred to as a "non-gravitating" or "stealth" scalar field. Two examples of massive waves of a $\phi$-field coupled nonminimally to the Ricci curvature and with a potential are given in [29]; they achieve $T_{a b}^{(\phi)}=0$ and no other form of matter is present. As a result, the spacetime is Minkowskian (spherically symmetric and static), providing a non-trivial realization of "vacuum" and of version 2 of the Jebsen-Birkhoff theorem. Other examples of nongravitating matter distributions are given in Refs. 30].

In the language of field theory, the field content of General Relativity consists only of a massless spin two field and gravitational radiation is quadrupole to lowest order. Hence spherically symmetric sources cannot excite gravitational radiation and the gravitational charge (i.e., the mass-energy) of the source is conserved - the spacetime around a spherically symmetric source must be static.

It is usually remarked that the Jebsen-Birkhoff theorem allows, as a corollary, an extension to General Relativity of the iron sphere theorem of Newtonian gravity stating that the gravitational field of a spherically symmetric distribution of mass inside a spherical cavity is zero. In spherical symmetry, if the energy distribution inside a cavity is static, the solution of the Einstein equations will be static. In vacuo, it is Minkowski space, i.e., the Schwarzschild solution corresponding to zero mass. If matter inside the cavity consists only of a cosmological constant the interior solution is spatially homogeneous 
and isotropic about every point and, therefore, (anti-)de Sitter.

\section{THE JEBSEN-BIRKHOFF THEOREM IN SCALAR-TENSOR GRAVITY}

With the advent of the Jordan [31] and Brans-Dicke [32] theories first and of scalar-tensor theories later [33], the validity of the Jebsen-Birkhoff theorem was investigated in alternative gravity [34 39, 41 46].

In general, the theorem does not hold in scalar-tensor gravity: one needs to impose that the effective stressenergy tensor $T_{a b}^{(\phi)}$ of the Brans-Dicke-like scalar field of the theory is time-independent in order for the metric to be static (this requirement is usually achieved by imposing that $\phi$ is time-independent, but is also obtained by a stealth field $\phi[29,30])$. The failure of the theorem in the presence of time-dependent scalars opens the door for new phenomenology in scalar-tensor gravity which is unknown in General Relativity. The failure of the JebsenBirkhoff theorem is to be expected: since scalar-tensor gravity has a new spin zero degree of freedom in comparison with General Relativity, scalar monopole radiation can occur. In Einstein's theory monopole radiation is forbidden by the Jebsen-Birkhoff theorem, which is a consequence of the fact that the gravitational field is represented only by a spin two field. Since in Einstein's theory gravitational radiation is necessarily quadrupole to lowest order, spherically symmetric pulsating sources cannot generate gravitational radiation and the metric must be static. This is no longer true in scalar-tensor gravity, in which the time-varying monopole moment of a radially pulsating spherical source generates propagating scalar radiation which makes also the metric non-static.

Let us consider a scalar-tensor theory of gravity described by the action

$$
\begin{aligned}
S_{S T} & =\frac{1}{16 \pi} \int d^{4} x \sqrt{-g}\left[\phi R-\frac{\omega(\phi)}{\phi} g^{a b} \nabla_{a} \phi \nabla_{b} \phi-V(\phi)\right] \\
& +S^{(m)}
\end{aligned}
$$

The field equations can be written in the form of effective
Einstein equations as

$$
\begin{aligned}
R_{a b}-\frac{1}{2} g_{a b} R & =\frac{8 \pi}{\phi} T_{a b}^{(m)} \\
& +\frac{\omega(\phi)}{\phi^{2}}\left(\nabla_{a} \phi \nabla_{b} \phi-\frac{1}{2} g_{a b} \nabla^{c} \phi \nabla_{c} \phi\right) \\
& +\frac{1}{\phi}\left(\nabla_{a} \nabla_{b} \phi-g_{a b} \square \phi\right)-\frac{V(\phi)}{2 \phi} g_{a b} \\
& \equiv \frac{8 \pi}{\phi}\left(T_{a b}^{(m)}+T_{a b}^{(\phi)}\right), \\
(2 \omega+3) \square \phi & =8 \pi T^{(m)}-\frac{d \omega}{d \phi} \nabla^{c} \phi \nabla_{c} \phi+\phi \frac{d V}{d \phi}-2 V,
\end{aligned}
$$

where $T_{a b}^{(m)}$ is the matter energy-momentum tensor and we assume that $\phi>0$ in conjunction with $\omega>-3 / 2$ to guarantee the positivity of the effective gravitational coupling [47]

$$
G_{e f f}=\frac{2(\omega+2)}{2 \omega+3} \frac{1}{\phi} .
$$

In this form, it is easy to see that the scalar field $\phi$ acts as an effective form of matter in the field equations (17) and therefore, by imposing that the matter stress-energy tensor $T_{a b}^{(m)}$ vanishes, one is left with an effective stress-energy tensor $T_{a b}^{(\phi)}$ such that $T_{00}^{(\phi)}$ could be time-dependent and $T_{0 i}^{(\phi)} \neq 0$ if $\phi$ depends on time. In other words, a time-dependent Brans-Dicke-like field $\phi$ spoils the validity of the Jebsen-Birkhoff theorem and only the assumption that $\phi$ is time-independent (or that $T_{a b}^{(\phi)}$ is static, as for a time-dependent stealth field $\phi$ ) restores the staticity of a spherically symmetric solution. This can be checked explicitly using the field equations, which we do in the following.

\section{The trivial case $\phi=$ constant}

The case $\phi=$ const. $\equiv \phi_{0}>0$ is trivial and eq. (17) reduces to

$$
R_{a b}-\frac{1}{2} g_{a b} R=\frac{8 \pi}{\phi_{0}} T_{a b}^{(m)}-\frac{V_{0}}{2 \phi_{0}} g_{a b}
$$

where $V_{0} \equiv V\left(\phi_{0}\right)$, so that the theory degenerates to General Relativity with the cosmological constant $\Lambda \equiv$ $V_{0} /\left(2 \phi_{0}\right)$. If $T_{a b}^{(m)}$ is such that the energy distribution is static (including the case $T_{a b}^{(m)}=0$ ), version 1 of the Jebsen-Birkhoff theorem holds and the metric is static in the region in which the coordinate gradients preserve their causal character. The same happens if $T_{a b}^{(\phi)}$ vanishes with $\phi \neq$ const. 


\section{Static (but non-constant) Brans-Dicke-like field}

Let us assume that the spacetime metric which solves the field equations (17) and (18) is spherically symmetric with line element (11). Then

$$
\nabla^{c} \phi \nabla_{c} \phi=-\frac{\dot{\phi}^{2}}{A^{2}}+\frac{\phi^{\prime 2}}{B^{2}}
$$

the only non-vanishing Christoffel symbols are

$$
\begin{aligned}
& \Gamma_{00}^{0}=\frac{\dot{A}}{A}, \quad \Gamma_{01}^{0}=\Gamma_{10}^{0}=\frac{A^{\prime}}{A}, \quad \Gamma_{11}^{0}=\frac{B \dot{B}}{A^{2}} \\
& \Gamma_{00}^{1}=\frac{A A^{\prime}}{B^{2}}, \quad \Gamma_{01}^{1}=\Gamma_{10}^{1}=\frac{\dot{B}}{B}, \quad \Gamma_{11}^{1}=\frac{B^{\prime}}{B} \\
& \Gamma_{22}^{1}=-\frac{r}{B^{2}}, \quad \Gamma_{33}^{1}=-\frac{r}{B^{2}} \sin ^{2} \theta \\
& \Gamma_{12}^{2}=\Gamma_{21}^{2}=\frac{1}{r}, \quad \Gamma_{33}^{2}=-\sin \theta \cos \theta \\
& \Gamma_{13}^{3}=\Gamma_{31}^{3}=\frac{1}{r}, \quad \Gamma_{23}^{3}=\Gamma_{32}^{3}=\frac{\cos \theta}{\sin \theta}
\end{aligned}
$$

and the d'Alembertian of $\phi$ is

$$
\begin{aligned}
\square \phi & =-\frac{1}{A^{2}}\left(\ddot{\phi}-\frac{\dot{A}}{A} \dot{\phi}-\frac{A A^{\prime}}{B^{2}} \phi^{\prime}\right) \\
& +\frac{1}{B^{2}}\left(\phi^{\prime \prime}-\frac{B \dot{B}}{A^{2}} \dot{\phi}-\frac{B^{\prime}}{B} \phi^{\prime}\right)+\frac{2 \phi^{\prime}}{r B^{2}} .
\end{aligned}
$$

The $(0,1),(0,0)$, and $(1,1)$ components of the field equations (17) yield

$$
\begin{aligned}
& \frac{2 \dot{B}}{B r}=\frac{8 \pi}{\phi} T_{01}^{(m)}+\omega \frac{\dot{\phi} \phi^{\prime}}{\phi^{2}}+\frac{1}{\phi}\left(\dot{\phi}^{\prime}-\frac{A^{\prime}}{A} \dot{\phi}-\frac{\dot{B}}{B} \phi^{\prime}\right), \\
& A^{2}\left(\frac{1}{r^{2}}+\frac{2 B^{\prime}}{B^{3} r}-\frac{1}{B^{2} r^{2}}\right)=\frac{8 \pi}{\phi} T_{00}^{(m)} \\
& +\frac{\omega}{2 \phi^{2}}\left(\dot{\phi}^{2}+\frac{A^{2}}{B^{2}} \phi^{\prime 2}\right) \\
& +\frac{A^{2}}{B^{2} \phi}\left(\phi^{\prime \prime}-\frac{B \dot{B}}{A^{2}} \dot{\phi}-\frac{B^{\prime}}{B} \phi^{\prime}+\frac{2 \phi^{\prime}}{r}\right)+\frac{V A^{2}}{2 \phi}, \\
& \frac{2 A^{\prime}}{A r}-\frac{B^{2}}{r^{2}}+\frac{1}{r^{2}}=\frac{8 \pi}{\phi} T_{11}^{(m)}+\frac{\omega}{\phi^{2}}\left(\phi^{\prime 2}+\frac{B^{2}}{A^{2}} \dot{\phi}^{2}\right) \\
& +\frac{B^{2}}{A^{2} \phi}\left(\ddot{\phi}-\frac{\dot{A}}{A} \dot{\phi}-\frac{A A^{\prime}}{B^{2}} \phi^{\prime}-\frac{2 A^{2}}{B^{2} r} \phi^{\prime}\right)-\frac{V B^{2}}{2 \phi} .
\end{aligned}
$$

If there are no radial energy currents $\left(T_{01}^{(m)}=0\right)$ and, in particular, in vacuo $\left(T_{a b}^{(m)}=0\right)$ and assuming that $\phi$ is time-independent $(\phi=\phi(r))$, eq. (28) yields

$$
\frac{\dot{B}}{B}\left(\frac{2}{r}+\frac{\phi^{\prime}}{\phi}\right)=0,
$$

so that either $\dot{B}=0$ or $\phi(r)=C / r^{2}$, where $C>0$ is a constant. If $\phi=C / r^{2}$ then eq. (29) yields

$$
B^{2}=\frac{2 C(2 \omega+3)}{2 C+V r^{4}}
$$

in vacuo and $B=B(r)$, therefore we focus on the $\dot{B}=0$ situation. Eq. (30) reduces to

$$
\begin{aligned}
\frac{A^{\prime}}{A}\left(\frac{2}{r}+\frac{\phi^{\prime}}{\phi}\right) & =\frac{8 \pi}{\phi} T_{11}^{(m)}+\frac{B^{2}-1}{r^{2}}+\omega\left(\frac{\phi^{\prime}}{\phi}\right)^{2}-\frac{2}{r} \frac{\phi^{\prime}}{\phi} \\
& -\frac{B^{2} V}{2 \phi} .
\end{aligned}
$$

If $\partial T_{11}^{(m)} / \partial t=0$ (in particular in vacuo), the right hand side of this equation is $t$-independent, implying that $A$ can depend from $t$ at most through a multiplicative factor as in $A(t, r)=f(t) a(r)$. In this case a redefinition of the time coordinate $d \bar{t}=f(t) d t$ absorbs the time-dependent factor into $\bar{t}$ and the metric can be cast in locally static form. Then, the other field equations imply that also the radial pressures $T_{i i}^{(m)}(i=1,2,3)$ are time-independent.

The fact that the scalar field $\phi$ needs to be static or non-gravitating in order to rescue the Jebsen-Birkhoff theorem was established on several occasions for particular scalar-tensor theories. Schücking [34] derived the result for Jordan's theory, a precursor of Brans-Dicke theory [31]; Reddy [37] studied the electro-vacuum case of the Sen-Dunn theory [63] and of the conformally coupled scalar field [45] (the former was revisited in [41] and the latter in [42]; the Sen-Dunn theory was reconsidered, and some errors of [37] corrected, in [38]). Venkateswarlu and Reddy studied electrovacuum in more general scalartensor theories [46].

To summarize, when the Brans-Dicke-like scalar is static but non-constant, the solution of the field equations can be different from Schwarzschild-(anti)de Sitter. If this field is constant the theory reduces to General Relativity, for which the Jebsen-Birkhoff theorem holds. As a corollary, the spherically symmetric solution inside an empty cavity is static only if the scalar field $\phi$ is assumed to be static or non-gravitating (Minkowski space if there is no potential and (anti-)de Sitter space if the potential is non-zero).

\section{THE JEBSEN-BIRKHOFF THEOREM IN THE EINSTEIN FRAME REPRESENTATION OF SCALAR-TENSOR GRAVITY}

By performing the conformal transformation of the metric and redefining non-linearly the Brans-Dicke-like 
scalar as in

$$
\begin{aligned}
& g_{a b} \rightarrow \tilde{g}_{a b}=\Omega^{2} g_{a b}, \quad \Omega=\sqrt{G \phi}, \\
& d \tilde{\phi}=\sqrt{\frac{|2 \omega(\phi)+3|}{16 \pi G}} \frac{d \phi}{\phi}
\end{aligned}
$$

for $\omega \neq-3 / 2$, the scalar-tensor action (16) assumes the Einstein frame form

$$
\begin{aligned}
& S_{S T}=\int d^{4} x \sqrt{-\tilde{g}}\left[\frac{\tilde{R}}{16 \pi G}-\frac{1}{2} \tilde{g}^{a b} \tilde{\nabla}_{a} \tilde{\phi} \tilde{\nabla}_{b} \tilde{\phi}-U(\tilde{\phi})\right. \\
& \left.+\frac{\mathcal{L}^{(m)}}{(G \phi)^{2}}\right]
\end{aligned}
$$

where a tilde denotes quantities associated with the rescaled metric $\tilde{g}_{a b}, \mathcal{L}^{(m)}$ is the matter Lagrangian density, and

$$
U(\tilde{\phi})=\frac{V[\phi(\tilde{\phi})]}{[G \phi(\tilde{\phi})]^{2}}
$$

This is formally the action of General Relativity with a minimally coupled scalar field $\tilde{\phi}$ but with the important difference that this scalar now couples explicitly to matter and the units of time and length scale with $\Omega$, while the unit of mass scales as $\Omega^{-1}$ [48]. The Einstein frame field equations are

$$
\begin{aligned}
& \tilde{R}_{a b}-\frac{1}{2} \tilde{g}_{a b} \tilde{R}=\frac{8 \pi G}{(G \phi)^{2}} T_{a b}^{(m)}+8 \pi G \tilde{T}_{a b}^{(\tilde{\phi})}, \\
& \tilde{\square} \tilde{\phi}-\frac{d U}{d \tilde{\phi}}=\frac{8 \pi G T^{(m)}}{(G \phi)^{2}}
\end{aligned}
$$

whereas

$$
\tilde{T}_{a b}^{(\tilde{\phi})}=\tilde{\nabla}_{a} \tilde{\phi} \tilde{\nabla}_{b} \tilde{\phi}-\frac{1}{2} \tilde{g}_{a b} \tilde{g}^{c d} \tilde{\nabla}_{c} \tilde{\phi} \tilde{\nabla}_{d} \tilde{\phi}-\frac{U(\tilde{\phi})}{2} \tilde{g}_{a b}
$$

is the canonical stress-energy tensor for a scalar field minimally coupled with the curvature, which satisfies the weak energy condition if $V \geq 0$. If the metric $g_{a b}$ is of the spherically symmetric form (11) also the rescaled $\tilde{g}_{a b}$ assumes the same form with $\Omega=\Omega(\phi)=\Omega(t, r)$.

It is well known that, at the classical level, the Jordan and the Einstein conformal frames are equivalent descriptions of the same theory [48 50] when the conformal transformation is well-defined, and one can recover easily in the Einstein frame the results discussed in the previous sections. When the scalar field $\tilde{\phi}$ is constant (which only happens if its Jordan frame cousin $\phi$ is constant) then one obtains the same equations of motion as in General Relativity with a cosmological constant (if $U(\tilde{\phi}) \neq 0$, which is equivalent to $V(\phi) \neq 0$ and $\phi \neq 0$, as follows from eq. (37)), and version 1 of the JebsenBirkhoff theorem is recovered.

If $\phi$ is not constant but is assumed to be independent of the time coordinate, $\tilde{\phi}$ given by eq. (35) is static as well and $\Omega=\Omega(r)$. Then, introducing the rescaled fourvelocity $\tilde{u}^{a}=\frac{u^{a}}{\Omega}=\left(\tilde{A}^{-1}, 0,0,0,\right)$, it is

$$
\begin{aligned}
\rho[\tilde{\phi}] & \equiv \tilde{T}_{a b}^{(\tilde{\phi})} \tilde{u}^{a} \tilde{u}^{b}=\frac{\tilde{\phi}^{\prime 2}}{2 \tilde{B}^{2}}+\frac{U(\tilde{\phi})}{2}, \\
J_{(r)}[\tilde{\phi}] & \equiv-\tilde{T}_{a b}^{(\tilde{\phi})} \tilde{u}^{a} \tilde{e}_{(r)}^{b}=0, \\
P_{(r)}[\tilde{\phi}] & \equiv \tilde{T}_{a b}^{(\tilde{\phi})} \tilde{e}_{(r)}^{a} \tilde{e}_{(r)}^{b}=\frac{\tilde{\phi}^{\prime 2}}{2 \tilde{B}^{2}}-\frac{U(\tilde{\phi})}{2},
\end{aligned}
$$

in the Einstein frame. By assuming that $\phi$ (or, equivalently, $\tilde{\phi})$ is static, the effective energy distribution described by $\tilde{T}_{a b}^{(\tilde{\phi})}$ is static and, if $T_{a b}^{(m)}$ is static as well, version 1 of the Jebsen-Birkhoff theorem holds and the spherically symmetric solution of the field equations $g_{a b}$ is static in a region in which the coordinate gradients preserve their causal character.

In the case $\omega=-3 / 2$ the field $\tilde{\phi}$ is not defined but nothing forbids the use of $\left(\tilde{g}_{a b}, \phi\right)$ as Einstein frame variables. The action then becomes

$$
\begin{aligned}
& S_{(-3 / 2)}=\int d^{4} x \sqrt{-\tilde{g}}\left[\frac{\tilde{R}}{16 \pi G}+\frac{3}{2} \tilde{g}^{a b} \tilde{\nabla}_{a} \phi \tilde{\nabla}_{b} \phi-V(\phi)\right. \\
& \left.+\frac{\mathcal{L}^{(m)}}{(G \phi)^{2}}\right],
\end{aligned}
$$

in which $\phi$ is a phantom field with negative-definite kinetic energy, which implies a sign change in the first term on the right hand sides of eqs. (41) and (43). Again, the metric will be static only if $\phi$ and $T_{a b}^{(m)}$ are static and the theory reduces to General Relativity with a cosmological constant if $\phi$ is constant.

It must be emphasized that the equivalence between Jordan and Einstein frames holds only when the conformal transformation is well-defined and breaks down if $\phi \rightarrow 0^{+}$or $\phi \rightarrow+\infty$, which can happen approaching a black hole horizon. For example, the black hole solutions of Brans class I [19], those of Bekenstein [20], and those of Campanelli and Lousto [21] are spherically symmetric and static but they are not Schwarzschild. They cause an apparent contradiction with a theorem by Hawking which, loosely speaking, states that stationary black holes in Brans-Dicke theory are the same as the stationary black holes of General Relativity and employs the Einstein frame in its proof. This apparent contradiction has generated some confusion in the literature and is discussed in the next subsection. 


\section{Hawking's theorem and the Jebsen-Birkhoff theorem in Brans-Dicke gravity}

Hawking's theorem [22] states that a stationary spacetime containing a black hole is a solution of the BransDicke field equations (with $V=0$ ) if and only if it is a solution of the Einstein field equations, and therefore it must be axially symmetric or static. The theorem is usually taken to mean that Brans-Dicke black holes are exactly the same as those of General Relativity: this is an overstatement and in fact many solutions of scalartensor theories including, but not necessarily limited to, Brans-Dicke gravity are known which describe black holes with a static scalar field and do not coincide with the Schwarzschild metric. For these solutions the scalar field either goes to zero or diverges on an event or apparent horizon and this feature invalidates the proof of Hawking's theorem, as we shall see below. The most well known example is probably that of Brans' class I solutions given by [19]

$$
\begin{aligned}
& d s^{2}=-\left(\frac{1-\mu / r}{1+\mu / r}\right)^{2 / \lambda} d t^{2}+\left(1+\frac{\mu}{r}\right)^{4}\left(\frac{1-\mu / r}{1+\mu / r}\right)^{\frac{2(\lambda-C-2)}{\lambda}}\left(d r^{2}+r^{2} d \Omega_{2}^{2}\right), \\
& \phi(r)=\phi_{0}\left(\frac{1-\mu / r}{1+\mu / r}\right)^{C / \lambda},
\end{aligned}
$$

where $\mu, C, \phi_{0}$ and $\lambda$ are constants with

$$
\lambda^{2}=(C+1)^{2}-C\left(1-\frac{\omega C}{2}\right)>0 .
$$

Clearly, this metric is static and not the Schwarzschild solution while it satisfies the vacuum Brans-Dicke field equations with $V=0$ for $r>\mu$ (three other classes of spherically symmetric solutions were found by Brans [19], although they are not all independent from each other [51]). Note that for the positive values of $C$ and $\lambda$ usually considered, the Brans-Dicke scalar field goes to zero on the horizon.

Let us consider now the proof of Hawking's theorem, which is performed in the Einstein frame 22. Another theorem 52] states that a stationary black hole in General Relativity must be axisymmetric and have spherical topology and relies on the weak (or the null) energy condition being satisfied. Hawking's theorem extends this previous theorem to Brans-Dicke theory 22], aiming to prove that the scalar field is static. It is pointed out in 22] that the advantage of going to the Einstein frame is that the rescaled Brans-Dicke scalar

$$
\tilde{\phi}=\sqrt{\frac{|2 \omega+3|}{16 \pi G}} \ln \left(\frac{\phi}{\phi_{*}}\right)
$$

(obtained by integrating eq. (35), where $\phi_{*}$ is a constant) has canonical kinetic energy density and obeys the weak and null energy conditions. The assumption that spacetime is stationary then implies that it is also axially symmetric 52] and, therefore, there exist a timelike Killing field $t^{a}$ and a spacelike Killing field $\psi^{a}$ (outside the horizon) and the Einstein frame scalar $\tilde{\phi}$ must necessarily be constant along the orbits of $t^{a}$ and $\psi^{a}$ in order to respect these symmetries, hence $\partial^{a} \tilde{\phi}$ can only be spacelike or zero outside the horizon.

Consider now a 4-dimensional volume $\mathcal{V}$ bounded by two Cauchy hypersurfaces $\mathcal{S}$ and $\mathcal{S}^{\prime}$ at two consecutive instants of time, a portion of the black hole event horizon, and spatial infinity [22]: the Einstein frame equation of motion (39) in vacuo and with $V=0$ becomes $\tilde{\square} \tilde{\phi}=0$, where $\tilde{\square} \equiv \tilde{g}^{a b} \tilde{\nabla}_{a} \tilde{\nabla}_{b}$ is the Einstein frame d'Alembertian. Multiplying this equation by $\tilde{\phi}$, integrating over $\mathcal{V}$, and using the Gauss theorem and the identity $\tilde{\phi} \tilde{\square} \tilde{\phi}=\tilde{\nabla}^{c}\left(\tilde{\phi} \tilde{\nabla}_{c} \tilde{\phi}\right)-\tilde{\nabla}^{c} \tilde{\phi} \tilde{\nabla}_{c} \tilde{\phi}$, one obtains [22]

$$
\int_{\mathcal{V}} d^{4} x \tilde{g}^{a b} \tilde{\nabla}_{a} \tilde{\phi} \tilde{\nabla}_{b} \tilde{\phi}=\int_{\partial \mathcal{V}} d S^{c}\left(\tilde{\phi} \tilde{\nabla}_{c} \tilde{\phi}\right)
$$

The integral over the boundary $\partial \mathcal{V}$ on the right hand side is split into four contributions:

$$
\int_{\partial \mathcal{V}} d S^{c}\left(\tilde{\phi} \tilde{\nabla}_{c} \tilde{\phi}\right)=\left(\int_{\mathcal{S}}+\int_{\mathcal{S}^{\prime}}+\int_{r=+\infty}+\int_{\text {horizon }}\right) d S^{c}\left(\tilde{\phi} \tilde{\nabla}_{c} \tilde{\phi}\right)
$$


The contributions from the portions of the Cauchy hypersurfaces $\mathcal{S}$ and $\mathcal{S}^{\prime}$ cancel out because they have the same absolute value due to the time symmetry but opposite signs because of the opposite directions of the outgoing unit normal on these hypersurfaces. The contribution from spatial infinity vanishes because $\tilde{\phi}$ vanishes there, whereas the contribution from the integral over the portion of the horizon is supposed to vanish because the projection of $\partial^{a} \tilde{\phi}$ along the null vector tangent to the horizon, which is a linear combination of $t^{a}$ and $\psi^{a}$, vanishes due to the symmetries 22]. This point is crucial: the argument is not valid in general because the Einstein frame scalar $\tilde{\phi}$ may not be defined at the horizon and, indeed, this is the case for the Brans class I solutions (45) and (46) in which the Einstein frame scalar $\tilde{\phi} \propto \ln \phi$ diverges on the horizon because the Jordan frame $\phi \rightarrow 0^{+}$there. The conformal transformation to the Einstein frame becomes ill-defined at the horizon and its variables $\left(\tilde{g}_{a b}, \tilde{\phi}\right)$ cannot be used on this surface. Of course, nothing forbids to use the Jordan frame instead of the Einstein one, but then the scalar $\phi$ violates the weak and null energy conditions because its stress-energy tensor given by eq. (17) has a non-canonical structure containing second derivatives of $\phi$ instead of being quadratic in the first derivatives (indeed, it is now well known that nonminimally coupled scalar fields can violate all the energy conditions [53]).

The scalar field in the known solutions violating the Hawking theorem does turn out to be static (which is what [22] intended to prove), but it has a radial dependence and these solutions do not coincide with the Schwarzschild metric.

Campanelli and Lousto [21] report static solutions of Brans-Dicke theory which possess a static but radiallydependent scalar. These authors comment on the violation of the null energy condition in the Jordan frame being the cause of the violation of the no-hair theorem. However, this comment (echoed in 51]) appears to be a bit misleading because Hawking's theorem discusses the Einstein frame scalar $\tilde{\phi}$ which does satisfy the energy condition, while Campanelli and Lousto refer to the Jordan frame $\phi$ which doesn't. Their comment is technically correct but, per se, does not help explaining the violation of Hawking's theorem. Note that, if the Brans-Dicke scalar $\phi$ does not go to zero or diverges on the horizon, Hawking's theorem applies and the solution is forced to be Schwarzschild.

\section{THE JEBSEN-BIRKHOFF THEOREM IN $f(R)$ GRAVITY}

We are now ready to come back to $f(R)$ gravity, which is the original motivation for our work, even though the understanding of the Jebsen-Birkhoff theorem and spherical symmetry in scalar-tensor gravity has merit in itself. The formal equivalence of $f(R)$ theories with scalar-tensor gravity has beeen discovered and rediscovered many times [18]. Metric $f(R)$ gravity is equivalent to a Brans-Dicke theory with Brans-Dicke parameter $\omega=0$ and a non-trivial potential, while Palatini $f(R)$ gravity is equivalent to an $\omega=-3 / 2$ Brans-Dicke theory with potential (see [7] for details). In the Palatini version, the scalar $\phi$ is non-dynamical, as has been pointed out in various works 7, 54, 56, 57]. The metric-affine version of $f(R)$ gravity [6] is not equivalent to a scalar-tensor theory and will not be considered here.

\section{Palatini $f(R)$ gravity}

Let us consider now the $\omega=-3 / 2, V \neq 0$ Brans-Dicke equivalent of Palatini $f(R)$ gravity. In vacuo, electrovacuo, or in any region in which the trace of the matter energy-momentum $T^{(m)}$ is constant, the d'Alembertian disappears from the field equation (18), which reduces to

$$
8 \pi G T^{(m)}+\phi \frac{d V}{d \phi}-2 V(\phi)=0
$$

no longer a differential but an algebraic or trascendental equation. If eq. (51) has solutions they are of the form $\phi=$ const. $\equiv \phi_{0}$. In this case the field equation (17) reduces to

$$
R_{a b}-\frac{1}{2} g_{a b} R=\frac{8 \pi}{\phi_{0}} T_{a b}^{(m)}-\frac{V\left(\phi_{0}\right)}{2 \phi_{0}} g_{a b},
$$

which describes General Relativity with a cosmological constant $\Lambda=\frac{V\left(\phi_{0}\right)}{2 \phi_{0}}$, for which Birkhoff's theorem holds if the matter distribution described by $T_{a b}^{(m)}$ is static (including the vacuum case $T_{a b}^{(m)}=0$ ) which is consistent, of course, with the previous assumption $T^{(m)}=$ const.

To summarize, the Jebsen-Birkhoff theorem holds in Palatini $f(R)$ gravity with a static matter distribution; the fact that the Jebsen-Birkhoff theorem holds when $T_{a b}^{(m)}=0$ is well known [7] and is due to the nondynamical nature of the Brans-Dicke scalar present in this class of theories, which acts as an effective form of matter without dynamics (this feature has been studied in detail, see the discussion and references in [7]).

\section{Metric $f(R)$ gravity}

Metric $f(R)$ gravity is equivalent to an $\omega=0$ BransDicke theory with a non-trivial potential. This time, in vacuo, the field equation (18) reduces to

$$
\square \phi=\frac{1}{3}\left[\phi \frac{d V}{d \phi}-2 V(\phi)\right],
$$

which is now a true dynamical equation. Since $\phi$ is dynamical and, in general, time-dependent the JebsenBirkhoff theorem is not valid, which has been noted on 
several occasions (see [7] and the references therein). Most studies in the literature impose the condition $\phi=$ const. (equivalent to $R=$ const. in the original $f(R)$ theory) for ease of calculation, and compare static solutions with Solar System experiments. This point of view carries the risk of not exploring the richer variety of solutions with $\partial \phi / \partial t \neq 0$, which are certainly more generic than static ones.

\section{CONCLUSIONS}

Motivated by the recent attention to metric and Palatini $f(R)$ gravity theories revived to explain the cosmic acceleration without dark energy, we have considered spherical symmetry and the Jebsen-Birkhoff theorem in these theories and, by extension, in general scalar-tensor gravity.

Generalizing the Jebsen-Birkhoff theorem of General Relativity to situations with matter present allows one to understand the validity, or lack thereof, of this theorem in scalar-tensor gravity because the scalar-tensor field equations can be rewritten as effective Einstein equations with the Brans-Dicke like scalar acting as a form of effective matter. Using the Jordan frame description of scalar-tensor gravity, this effective matter distribution must be static in order for the Jebsen-Birkhoff theorem to be valid. This conclusion is not hard to obtain but is seems that it is necessary to formulate it explicitly in order to make progress with $f(R)$ gravity. The situation can be summarized as follows: if $\phi$ is static the spherically symmetric solution is locally static between horizons but not necessarily Schwarzschild-(anti-)de Sitter; if $\phi$ is constant the solutions is Schwarzschild-(anti-)de Sitter.

Since scalar-tensor gravity admits an Einstein frame description in which the scalar has canonical form except for the fact that it couples directly to matter, the result obtained in the Jordan frame must be recovered in the Einstein frame description, and we checked that this is indeed the case. The equivalence (at the classical level) between Jordan and Einstein frame breaks down when the conformal transformation (34) and (35) becomes illdefined, and this occurrence allows one to understand the apparent contradiction between certain spherical solutions and Hawking's theorem on Brans-Dicke black holes. Shedding light onto this riddle certainly does not have deep new consequences (these non-Schwarzschild static solutions have now been known for a long time) but we are not aware of an explicit explanation in these terms in the literature.

Once the role of the Jebsen-Birkhoff theorem in scalartensor gravity is established, it is straightforward to understand that the validity of this theorem in Palatini $f(R)$ gravity is yet another manifestation of the nondynamical character of the Brans-Dicke scalar present in this theory. Similarly, the failure of the theorem in metric $f(R)$ gravity reflects the dynamical nature of the scalar degree of freedom present in these theories. Most current studies of spherically symmetric solutions in metric $f(R)$ gravity focus on static solutions missing time-dependent solutions which are, without doubt, more generic than static ones (although there is at present no mathematically well-defined meaning of "generic"). To complicate the issue, metric $f(R)$ theories of current interest are designed to produce an effective timevarying cosmological constant in order to explain the present acceleration of the universe without dark energy, and it is expected that "generic" solutions (if a meaning can be assigned to this adjective) will be asymptotically Friedmann-Lemaitre-Robertson-Walker solutions violating the Jebsen-Birkhoff theorem. Some solutions of this kind are known in General Relativity 28, 58], scalartensor gravity [59] and in metric $f(R)$ gravity [60], but they are still not understood very well even in the context of General Relativity and it will be interesting to study them further in the future.

It is a pleasure to thank Vincenzo Vitagliano for a discussion and the Natural Sciences and Engineering Research Council of Canada (NSERC) for financial support.

* vfaraoni@ubishops.ca

[1] A.G. Riess et al., Astron. J. 116, 1009 (1008); Astron. J. 118, 2668 (1999); Astrophys. J. 560, 49 (2001); Astrophys. J. 607, 665 (2004); S. Perlmutter et al., Nature 391, 51 (1998); Astrophys. J. 517, 565 (1999); J.L. Tonry et al., Astrophys. J. 594, 1 (2003); R. Knop et al., Astrophys. J. 598, 102 (2003); B. Barris et al., Astrophys. J. 602, 571 (2004).

[2] E.V. Linder, Am. J. Phys. 76, 197 (2008).

[3] S. Capozziello, Int. J. Mod. Phys. D 11, 483 (2002); S. Capozziello, S. Carloni, and A. Troisi, arXiv:astro-ph/0303041

[4] S.M. Carroll, V. Duvvuri, M. Trodden, and M.S. Turner, Phys. Rev. D 70, 043528 (2004).

[5] D.N. Vollick, Phys. Rev. D 68, 063510 (2003).

[6] T.P. Sotiriou, Class. Quantum Grav. 23, 5117 (2006); arXiv:gr-qc/0611158; arXiv:0710.4438, T.P. Sotiriou and S. Liberati, Ann. Phys. (NY) 322, 935 (2007); J. Phys. Conf. Ser. 68, 012022 (2008).

[7] T.P. Sotiriou and V. Faraoni, arXiv:0805.1726, to appear in Rev. Mod. Phys.

[8] S. Nojiri and S.D. Odintsov, Int. J. Geom. Meth. Mod. Phys. 4, 115 (2007); S. Capozziello and M. Francaviglia, Gen. Relat. Grav. 40, 357 (2008); N. Straumann, arXiv:0809.5148 H.-J. Schmidt, Int. J. Geom. Meth. Phys. 4, 209 (2007); V. Faraoni, arXiv:0810.2602 T.P. Sotiriou, J. Phys. Conf. Ser. 189, 012039 (2009); S. Capozziello, M. De Laurentis, and V. Faraoni, arXiv:0909.4672

[9] R.M. Wald, General Relativity (Chicago University Press, Chicago 1984).

[10] C.M. Will, Theory and Experiment in Gravitational Physics (CUP, Cambridge 1993). 
[11] F. Briscese, E. Elizalde, S. Nojiri and S. D. Odintsov, Phys. Lett. B 646, 105 (2007); A.V. Frolov, Phys. Rev. Lett. 101, 061103 (2008); A.A. Appleby and R.A. Battye, Phys. Lett. B 654, 7 (2008); S. Nojiri and S.D. Odintsov, Phys. Rev. D 78, 046006 (2008).

[12] K. Schleich and D.M. Witt, arXiv:0908.4110, arXiv:0910.5194.

[13] G.D. Birkhoff, Relativity and Modern Physics (Harvard Univ. Press, Cambridge, USA 1923).

[14] S. Deser and J. Franklin, Am. J. Phys. 73, 261 (2005).

[15] N.V. Johansen and F. Ravndal, Gen. Relat. Grav. 38, 537 (2006).

[16] S. Deser, Gen. Relat. Grav. 37, 2251 (2005).

[17] J.T. Jebsen, Ark. Mat. Ast. Fys. (Stockholm) 15, nr. 18 (1921), reprinted in Gen. Relat. Grav. 37, 2253 (2005).

[18] P.W. Higgs, Nuovo Cimento 11, 816 (1959); P. Teyssandier and P. Tourrenc, J. Math. Phys. 24, 2793 (1983); B. Whitt, Phys. Lett. B 145, 176 (1984); J.D. Barrow, Nucl. Phys. B 296, 697 (1988); J.D. Barrow and S. Cotsakis, Phys. Lett. B 214, 515 (1988); D. Wands, Class. Quantum Grav. 11, 269 (1994); T. Chiba, Phys. Lett. B 575, 1 (2005).

[19] C.H. Brans, Phys. Rev. 125, 2194 (1962).

[20] J.D. Bekenstein, Ann. Phys. 82, 535 (1974).

[21] M. Campanelli and C. Lousto, Int. J. Mod. Phys. D 2, 451 (1993).

[22] S.W. Hawking, Comm. Math. Phys. 25, 167 (1972).

[23] L.D. Landau and E.M. Lifschitz, The Classical Theory of Fields (Pergamon Press, Oxford 1989).

[24] J. Ehlers and A. Krasinski, Gen. Relat. Grav. 38, 1329 (2006).

[25] H. Stephani, D. Kramer, M. MacCallum, C. Hoenselaers, and E. Herlt, Exact Solutions of the Einstein Field Equations (CUP, Cambridge 2003).

[26] V.P Frolov and I.D. Novikov, Black Hole Physics (Kluwer Academic, Dordrecht 1998).

[27] A. Das, Prog. Theor. Phys. 24, 915 (1960).

[28] G.C. McVittie, Mon. Not. R. Astron. Soc. 93, 325 (1933).

[29] E. Ayon-Beato, C. Martinez, R. Troncoso, and J. Zanelli, Phys. Rev. D 71, 104037 (2005).

[30] L.M. Sokolowski, Acta Phys. Polon. 35, 587 (2004); E. Ayon-Beato, C. Martinez, and J. Zanelli, Gen. Relat. Grav. 38, 145 (2006); D.C. Robinson, Gen. Relat. Grav. 38, 153 (2006); D.A. Demir and B. Pulice, Phys. Lett. B 638, 1 (2006); N. Banerjee, R.K. Jain, and D.P. Jaktar, Gen. Relat. Grav. 40, 93 (2008).

[31] P. Jordan, Schwerkraft und Weltall (F. Vieweg \& Sohn, Braunschweig 1952).

[32] C.H. Brans and R.H. Dicke, Phys. Rev. 124, 925 (1961).

[33] P.G. Bergmann, Int. J. Theor. Phys. 1, 25 (1968); R.V. Wagoner, Phys. Rev. D 1, 3209 (1970); K. Nordvedt, Astrophys. J. 161, 1059 (1970).

[34] E. Schücking, Z. Phys. 148, 72 (1957).

[35] J. O'Hanlon and B.O.J. Tupper, Nuovo Cimento 7B, 305 (1972).

[36] D.R.K. Reddy, J. Phys. A 6, 1867 (1973).

[37] D.R.K. Reddy, J. Phys. A 10, 185 (1977).

[38] K.D. Krori and D. Nandy, J. Phys. A 10, 993 (1977).

[39] T. Singh and L.N. Rai, Indian J. Pure Appl. Math. 10, 1432 (1979).
[40] D.K. Sen and K.A. Dunn, J. Math. Phys. 12, 578 (1971).

[41] S.B. Dutta Choudhury and D. Battacharyya, J. Phys. A 13, 3643 (1980).

[42] T. Singh, Phys. Rev. D 34, 646 (1986).

[43] T. Singh, J. Math. Phys. 27, 1074 (1986).

[44] D.R.K. Reddy, M.B. Avadhanulu, and R. Venkateswarlu, Astrophys. Sp. Sci. 134, 201 (1987).

[45] D.R.K. Reddy, Astrophys. Sp. Sci. 140, 85 (1988).

[46] R. Venkateswarlu and D.R.K. Reddy, Astrophys. Sp. Sci. 159, 173 (1989).

[47] K. Nordtvedt, Phys. Rev. D 169, 1017 (1968).

[48] R.H. Dicke, Phys. Rev. 125, 2163 (1962).

[49] E.E. Flanagan, Class. Quantum Grav. 21, 417 (2004).

[50] V. Faraoni and S. Nadeau, Phys. Rev. D 75, 023501 (2007).

[51] A. Bhadra and K. Sarkar, Gen. Relat. Grav. 37, 2189 (2005).

[52] S.W. Hawking, Comm. Math. Phys. 25, 152 (1972).

[53] J.D. Bekenstein, Phys. Rev. D 11, 2072 (1975); L.H. Ford, Phys. Rev. D 35, 2339 (1987); M. Madsen, Class. Quant. Grav. 5, 627 (1988); W.A. Hiscock, Class. Quant. Grav. 7, L35 (1990); C. Barcelo and M. Visser, Class. Quant. Grav. 17, 3843 (2000); L.H. Ford and T.A. Roman, Phys. Rev. D 64, 024023 (2001).

[54] E. Barausse, T.P. Sotiriou, and J.D. Miller, Class. Quantum Grav. 25062001 (2008); 25, 105008 (2008); EAS Publ. Ser. 30, 189 (2008).

[55] A. Iglesias, N. Kaloper, A. Padilla, and M. Park, Phys. Rev. D 76, 104001 ((2007).

[56] V. Faraoni, Phys. Lett. B 665, 135 (2008).

[57] N. Lanahan-Tremblay and V. Faraoni, Class. Quantum Grav. 24, 5667 (2007); V. Faraoni and N. LanahanTremblay, Phys. Rev. D 78, 064017 (2008).

[58] J. Sultana and C.C. Dyer, Gen. Relat. Grav. 37, 1349 (2005); V. Faraoni, Phys. Rev. D 80, 044013 (2009); M.L. McClure and C.C. Dyer, Class. Quantum Grav. 23, 1971 (2006); Gen. Relat. Grav. 38, 1347 (2006); V. Faraoni and A. Jacques, Phys. Rev. D 76, 063510 (2007); H. Saida, T. Harada, and H. Maeda, Class. Quantum Grav. 24, 4711 (2007); C. Gao, X. Chen, V. Faraoni, and YG. Shen, Phys. Rev. D 78, 024008 (2008); M. Nozawa and H. Maeda, Class. Quantum Grav. 25, 055009 (2008); H. Maeda, T. Harada, and B.J. Carr, Phys. Rev. D 77, 024023 (2008); V. Faraoni, C. Gao, X. Chen, and Y.-G. Shen, Phys. Lett. B 671, 7 (2009).

[59] T. Clifton, D.F. Mota and J.D. Barrow, Mon. Not. R. Astr. Soc. 358, 601 (2005).

[60] T. Clifton, Class. Quantum Grav. 23, 7445 (2006); V. Faraoni, Class. Quantum Grav. 26, 195013 (2009).

[61] Alternatively, one can require that the Lie derivatives of $T_{a b}^{(m)}$ along the directions of the two Killing vectors $t^{a}$ (timelike) and $\psi^{c}$ (spacelike) vanish [12].

[62] The local nature of the Jebsen-Birkhoff theorem is emphasized in [12].

[63] The Sen-Dunn theory [40] is a scalar-tensor theory in which the combination of second derivatives $\nabla_{a} \nabla_{b} \phi-$ $g_{a b} \square \phi$ on the right hand side of the field equations (17) vanishes identically, making a massless $\phi$ satisfy the weak energy condition in the Jordan frame. 